\title{
El manejo del agua en Honduras
}

Ciencia y Tecnología (CT) entrevista al Doctor Rodolfo Ochoa Álvarez, Ingeniero Civil, con una Maestría en Ingeniería Ambiental, especialista en Hidrogeología, y actualmente es Director y Asesor Técnico de Investigación y Análisis Técnico en Agua y Saneamiento (sectorial) - Servicio Autónomo Nacional de Acueductos y Alcantarillados (SANAA) desde 1998. En su larga trayectoria como investigador, tiene en su haber varias publicaciones y participaciones, así mismo ha sido miembro fundador del Grupo interinstitucional de Agua Potable y Saneamiento en Honduras, ahora constituido en Red, Miembro fundador de la plataforma del Agua de Honduras (PAH), Miembro fundador de la Comisión Nacional de Biodiversidad, Miembro de la Asociación Hondureña de Ingeniería Sanitaria (AHIDIS) y Miembro fundador de laAsociación Nacional del Agua GWP-Honduras.

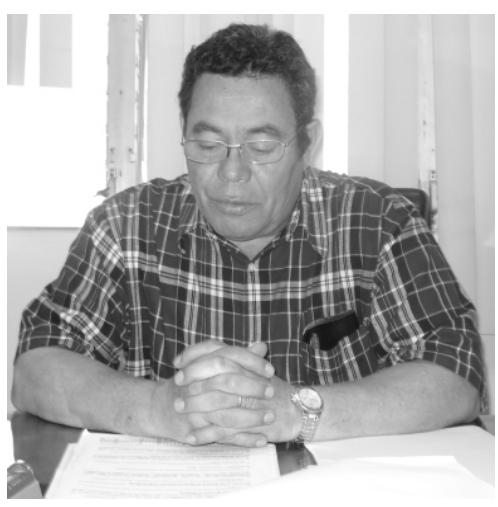

Rodolfo Ochoa Álvarez

\section{CT/ ¿Cómo resumiría el problema del agua en nuestro país?}

$\mathrm{R} /$. El problema del agua podríamos resumirlo en una palabra: descoordinación. ¿Por qué descoordinación? Para que exista una adecuada gestión integral de agua potable y saneamiento, lo que nosotros denominamos GIAPS, es necesario que trabajen de la mano el Estado, las Municipalidades y la Sociedad Civil. Por lo pronto hay un acercamiento bastante grande entre la sociedad civil e instituciones como el SANAA, debido a que existe un programa de apoyo rural en el cual está el acercamiento entre estas dos partes; sin embargo, otros arreglos entre el Estado y las Municipalidades escapan al ámbito de coordinación con el SANAA y no hemos podido encontrar una forma de coordinar las necesidades municipales con la estrategia de gobierno y con la responsabilidad técnica existente. Esto se agrava un poco más, hablando de sociedad civil, en el sentido de que existe una gran cantidad de organizaciones no gubernamentales y organizaciones privadas de desarrollo, que tampoco tienen una coordinación entre sí, como sectores independientes. Se podría decir que existe buena intención y eficiencia en los programas propios que se desarrollan, pero el efecto multiplicador no se nota por esa falta de coordinación. 


\section{$\mathrm{CT} / ¿$ Cuántas cuencas hidrográficas existen en el país y cuáles están protegidas?}

R/. En este caso hay que diferenciar algunos conceptos: cuenca hidrográfica está íntimamente relacionada con el agua; el agua que llueve escurre a través de un terreno limitado por el parte aguas o sea por las montañas que circunda un rio, y esta agua llega finalmente al mar. Entonces una estrategia que considere el desarrollo desde la montaña hasta el mar, es una forma de actuar. La otra es, si el concepto de cuenca lo estamos considerando como áreas protegidas, en este caso sería cuenca alta, cuencas forestales le llamamos nosotros; el término todavía no ha sido consensuado, pero sí estamos de acuerdo que el sector forestal trabaja principalmente en las micro cuencas, que formalmente se encuentran en la parte alta de las cuencas hidrográficas. Diferenciando estos conceptos, podríamos decir que existen 21 cuencas hidrográficas en el país, incluyendo las del Atlántico y las islas del Pacífico, y como áreas protegidas de micro cuencas o cuenca alta, hay 89 áreas protegidas.

\section{$\mathrm{CT} /$ : A nivel estatal ¿qué acciones se están ejecutando y qué nivel de éxito se ha tenido?}

R/. Para poder desarrollar una adecuada gestión, no solamente de agua potable y saneamiento, sino de la gestión integral de los recursos hídricos, (GIRH) entendiendo esto como otras áreas de acción del agua, como son: riego, energía eléctrica, recreación, inclusive la parte de utilización del agua como transporte, caso Islas de la Bahía o las costas del país, es necesaria una coordinación más extensa incluyendo agua potable y saneamiento, porque en la GIRH, entran una serie de instituciones, y para poder regular adecuadamente el papel que les corresponde y la realización de acciones efectivas, es necesaria una nueva Ley General del Agua. La actual ley data del año 1927 según la cual uno de los usos principales del agua, era para el ferrocarril; eso nos demuestra lo obsoleto de la ley actual. En este orden, en Honduras cualquiera hace lo que quiere con el agua y con sus cursos y por eso, uno de los elementos básicos es desarrollar una nueva legislación al respecto. Desde el punto de vista del agua potable y saneamiento, se ha observado que existen leyes conexas como la del ambiente, la de las municipalidades, pero también la del ordenamiento territorial, la ley forestal, la ley de agua potable y saneamiento, y la ley general, que son las que conforman el entorno del conocimiento, regulación del recurso hídrico y su utilización. En ese sentido ya tenemos la Ley Forestal, la Ley de Agua Potable y Saneamiento, la Ley de Ordenamiento Territorial y la única que hace falta es la Ley General del Agua, 
que en estos momentos se encuentra en segundo dictamen en el Congreso Nacional.

\section{CT/ En Honduras ¿cuáles instituciones trabajan en la gestión del agua en cuencas hidrográficas?}

R/. Nuevamente tenemos que revisar la conceptualización. Como "cuencas hidrográficas" trabajan la Secretaría de Agricultura, la SERNA (Secretaría del Ambiente y Recursos Naturales), un programa que trabaja junto con SETCO y Agricultura denominado Forcuencas, también un proyecto que aun está vigente y es el programa Mira de USAID. Contamos con iniciativas locales como Amu Prolago, El Guizayote, el proyecto de El Cajón, Guacerique y Concepción, Zamorano y otros proyectos como Lempira Sur, que consideran la cuenca hidrográfica como unidad de planificación. Como "áreas protegidas" o como "micro cuencas", definitivamente es una actividad propia del Instituto Forestal.

\section{CT/ ¿Qué programas existen para la protección de cuencas hidrográficas?}

R/. Para cuencas hidrográficas, el Programa de Manejo de Recursos Naturales (MARENA), que corresponde al Programa Nacional de Desarrollo Rural, PRONADER, FORCUENCAS y MIRA son los más fuertes.

\section{CT/ En lo social ¿qué nivel de involucramiento tienen las comunidades y cuáles han sido las experiencias de mayor éxito?}

R/. Quiero mencionarle que la Ley Marco de Agua Potable y Saneamiento, establece como uno de los cambios en el manejo de los recursos del agua, un comité de protección de micro cuencas dentro de las juntas de agua y saneamiento. Sabemos que esto va caminando lento pero ya hay bastantes comunidades que tienen conformadas esta unidad de protección de micro cuencas, y existen otros niveles manejados por ONGs, principalmente en las áreas protegidas que mencionábamos. Cerca de Tegucigalpa, un ejemplo de ello es AMITRIGRA, que protege la montaña de La Tigra de donde se obtiene un tercio del agua para Tegucigalpa, a través de 21 manantiales que los están protegiendo correctamente. A nivel de cuenca hidrográfica podríamos mencionar el programa del Lago de Yojoa donde se han estado reuniendo todas las municipalidades que tienen relación con el agua, y siendo una cuenca hidrográfica endógena, se están haciendo los estudios en forma completa. 


\section{$\mathrm{CT} /$ ¿Cuáles son las acciones que como país se tienen para la gestión integral de los ríos, acuíferos y los recursos hídricos en general?}

$\mathrm{R}$. Bueno, la protección de los ríos es una actividad que le corresponde a la SERNA y el programa que ellos están desarrollando e impulsando en estos momentos, es la protección de la ribera de los ríos, a través de un programa que se denomina "Adoptemos un Río" y lo promueven en ferias del agua. Sin embargo a nivel de cuenca hidrográfica todavía no se tiene una estrategia, sólo lo que hemos mencionado en algunos programas donde el fin va más enfocado a la generación de proyectos que a la protección sostenida del recurso. Yo creo que en Honduras estamos todavía en pañales, a pesar de que se ha generado información básica de uso a nivel mesoamericano. Hace 10 años Honduras iba de Costa Rica y en el último quinquenio, El Salvador, Guatemala y Nicaragua se nos han adelantado en recursos hídricos al tener bien diferenciado el rol de las secretarias del ambiente respecto a las de Recursos Naturales.

Sí ha habido logros; uno de ellos es haber participado en la formación de diferentes redes que están funcionando y haciendo alguna actividad que ayuda al desarrollo de los recursos hídricos. Podemos mencionar la Plataforma del Agua de Honduras, la Asociación Nacional de Agua de Honduras (GWP), la Red de Agua y Saneamiento de Honduras (RAS-HON), la Asociación de Juntas de Agua (AHJASA), y todas ellas actúan, desde su ángulo, a nivel de capacitación rural como la AHJASA. La RASHON trabaja en transferencia de tecnología de otros países hacia Honduras, pero su visión está bastante enfocada en el manejo a través de organizaciones no gubernamentales.

La Plataforma de Agua de Honduras, igual que GWP, tienen una visión de desarrollar una gestión integral de los recursos hídricos, pero está emergiendo en estos momentos; apenas se tiene en GWP-Honduras (la Asociación Nacional de Agua de Honduras), la planificación centroamericana, y de allí sabremos qué acciones vamos a desarrollar a nivel de país.

\section{CT/ ¿Cómo valora la normatividad existente en materia de agua? ¿Es suficiente?, ¿Es la adecuada?}

$\mathrm{R} /$. En cuanto a la normatividad, podemos decir que no es mucha pero tampoco es poca. La normatividad es difícil de evaluar si es suficiente o no, porque esto depende del desarrollo, depende de país a país, depende de las necesidades y la normatividad cambia inclusive de acuerdo al mismo desarrollo. En Honduras 


\section{CT/ ¿Existe alguna investigación que nos hable de los ciclos de sequía en el país?}

R/. No conozco que se haya realizado fuera del SANAA algún acercamiento al respecto. Yo le puedo mencionar que existe una ficha técnica dentro del SANAA elaborada por la DIAT (División de Investigación de Análisis Técnico), que revisa la influencia del Fenómeno de El Niño, en la que se puede observar una periodicidad en la manifestación de este tipo de eventos. Y nos hemos extendido un poco más allá al analizar la periodicidad del fenómeno de La Niña y sus ciclos, que podrían llamarse multiciclos, porque la periodicidad puede ser en años, en decenas de años o en cientos y miles de años, pues hay una ciclisidad permanente en el asunto del agua, y hemos investigado vía internet y en entrevistas con amigos, algunas informaciones que hemos plasmado en fichas técnicas y que están disponibles para el público.

\section{CT/ En los distritos de riego, ¿existe un plan integral para manejar el recurso agua?}

$\mathrm{R} /$. Sí existen planes integrales, aunque no en forma sistemática; más bien como una iniciativa o una necesidad que se siente entre las juntas de regantes. Platicando con un amigo de la Secretaria de Agricultura y Ganadería, me informaba que existen 6 regiones 0 zonas en las que están trabajando, y cuando existe necesidad de compaginar o utilizar el agua de riego para uso potable, se ponen de acuerdo y generan la reglamentación del uso que le van a dar.

\section{CT/ ¿Existe un sistema de alerta hidrometeorológica y cuáles son sus principales componentes?}

R/. A raíz del huracán Mitch, el servicio geológico norteamericano apoyó a Honduras con tecnología adecuada para poder prever a tiempo los desastres que pudiesen ocasionarse por eventos meteorológicos, y comenzaron a apoyar a la SERNA, al SANAA y otras instituciones en la colocación de estaciones telemétricas. Existen actualmente 75 estaciones telemétricas funcionando a nivel nacional en 15 de las 21 cuencas hidrográficas. La mayor parte de ellas tienen estaciones en cuenca alta, cuenca media y cuenca baja, con lo cual se puede dar seguimiento a las crecidas de los ríos, desde diferentes partes de un mismo río. El elemento principal que esto conlleva es una estación automática de medición computarizada en el margen de los ríos, que manda una señal a una torre, la que emite los datos a un ordenador central y este ordenador central los envía a la web. Contamos con una clave con la que puede ingresar cualquier persona a esta información, en tiempo real. 
mencionábamos que la falta que se nota como un agujero negro en este aspecto, es la ley general, porque ese es el marco que nos va a dar las directrices de cómo actuar en las diferentes instituciones, en su relación con el agua. Tenemos normativa de la calidad, tenemos normativa ambiental; respecto a la calidad del agua tenemos normativa de los desechos de las aguas residuales hacia los ríos y por lo pronto, eso es más que suficiente, porque, inclusive, al adoptarse algunos criterios usados en otros países con mayores posibilidades económicas, mucho del articulado que se ha desarrollado no lo podemos cumplir, no porque no se deba, 0 porque no sea necesario tenerlo, sino por las cuestiones económicas

\section{CT/ ¿Cómo visualiza el problema del agua en los próximos 20 años? ¿Mejoramos o empeoramos? ¿De qué depende esto?}

R/. Vamos mejorando en el sentido de ir aclarando los roles del Estado en cuanto al manejo del recurso hídrico, ya sea en vigilancia, en el uso o en la regulación. Ya hay instituciones que están trabajando al respecto, lo único que nos falta es la autoridad del agua y esta autoridad es la que está contemplada en esta ley general; por eso es que le doy tanta fuerza y he hablado tanto de la falta de la Ley General de Agua. Una de las situaciones que debemos revisar es si las acciones que estamos desarrollando, van a la par del crecimiento poblacional. El crecimiento poblacional nos absorbe y el caso patético es el de Tegucigalpa donde en el pasado hemos analizado 10 años atrás para satisfacer las necesidades del crecimiento poblacional, a este momento estamos haciendo varios proyectos que sostienen el problema, que no lo hacen crecer más, pero no lo resolvemos. Esto quiere decir que como política del agua, como legislación del agua, estamos débiles, aunque tenemos una fortaleza de gente pensando, gente actuando a nivel nacional pero trabajando independientemente cada uno en lo que mejor cree que debe hacer; al final posiblemente estamos perdiendo energía duplicando actividades, o en el mejor de los casos nuestras actividades son menos impactantes al trabajar solos que al trabajar en grupo. Mencionaba que las redes eran una punto muy importante en Honduras, pero son instituciones pensantes a mi criterio, no son actuantes. El trabajo de campo requiere de mayor número de personas resolviendo los problemas en cada una de las comunidades, y esa coordinación es necesaria para que tanto lo que tenemos en ley, que es bastante, y la capacidad instalada que es bastante, puedan conjuntarse y trabajar en una misma dirección, todos en función de un mismo plan general y no con los cientos de planes que tienen las cientos de ONGs trabajando en el país y decenas de instituciones de Gobierno quitándose unos y otros, financiamiento para proyectos. La otra visión que se tiene como problema del agua en los próximos 20 años es el cambio climático. Nosotros 
desarrollamos un seguimiento para observar lo que está sucediendo en estos momentos, y aunque creemos que estamos en un periodo largo de suficientes Iluvias, los fenómenos de El Niño se presentan sin aviso; son erráticos, se puede ver la ciclicidad que se ha investigado del pasado y puede ser en periodos de tres años, cuatro, cinco y hasta 7años, en que se vuelve a presentar. Esto no permite prepararse adecuadamente en una forma de actuación sistemática sino esperando que este año puede ser el quinto o el séptimo, y es hasta el momento en que se comienza a manifestar, que se hacen las actividades.

El plan económico es otro de los puntos que es importante mejorar debido a que todavía nuestros gobiernos trabajan con programas operativos anuales. Esto no permite ver el mediano ni el largo plazo en aspectos financieros; aunque las instituciones técnicas tengan una planificación de largo plazo, al final terminan adaptándose a una planificación económica del año. Esto ¿empeora o mejora? Yo me pondría en un punto neutro. Por suerte tenemos una suficiente capacidad instalada a nivel nacional, normativa que ya está casi en su proceso final de culminación y se debe luchar por esa coordinación necesaria. Lográndose esa coordinación, será un panorama alentador para el manejo de los recursos hídricos en el futuro del país. En resumen, es necesario que nuestro país cuente con tres instrumentos: La Ley General delAgua, Ordenamiento y coordinación institucional y Plan nacional de recursos hídricos con enfoque de Gestión Integral. 